P90 TOWARDS A PROTOCOL FOR THE MANAGEMENT OF VERY SEVERE CHRONIC LUNG DISEASE

M Hurley, R Khetan, J Bhatt. Nottingham Children's Hospital, Nottingham, UK

10.1136/thoraxjnl-2015-207770.227

Background While corticosteroids appear effective in reducing respiratory support requirements of babies with very severe chronic lung disease of prematurity (CLD), controversy remains regarding the most appropriate route, timing, preparation and dosage. Using Delphi methodology, consensus was reached involving 4-weekly pulses of methylprednisolone for 3 days at $500 \mathrm{mg} / \mathrm{m}^{2}$ in ventilator-dependent, or close to ventilation, babies in diffuse lung disease of childhood (Cunningham S., et al. Am J Respir Crit Care Med 189;2014:A4664). Use of hydroxychloroquine and azithromycin also reached consensus.

Aims and objectives To describe the features of those babies who received methylprednisolone, hydroxychloroquine and azithromycin ("consensus treatment") for severe CLD compared to those who did not in order to inform the drafting of a protocol. Methods A prospective database detailing care of babies with severe CLD referred to the CLD service at Nottingham Children's Hospital Jan 2009-Dec 2014 was used.

Results 147 children were referred to the service; 4 babies received consensus treatment. Those receiving consensus treatment were ventilated for longer 39(sd 4.6) versus $8(\mathrm{sd} 16.8$ ) days $(\mathrm{p}<0.001)$. Children receiving consensus treatment were significantly older at discharge $245(65)$ versus $95(45.5)$ days and were discharged with higher oxygen requirements $(1.0(0.4)$ vs. $0.3(0.2)$ litres).

Conclusions Babies with very severe CLD were successfully treated with consensus treatment. A change in practice toward discharge at higher oxygen delivery rates in such babies was safe. Further experience will refine the objective criteria for considering consensus treatment and would inform the design of a future randomised controlled trial.

\section{P91 POST-INFECTIVE OBLITERATIVE BRONCHIOLITIS ACQUIRED BEYOND THE FIRST 3 YEARS OF LIFE}

S Sonmez-Ajtai, S Moss. The Great North Children's Hospital, Newcastle Upon Tyne, UK

\subsection{6/thoraxjnl-2015-207770.228}

Introduction Obliterative bronchiolitis (OB) is a rare form of chronic obstructive lung disease that follows a severe insult to the lower respiratory tract, resulting in fibrosis of the small airways. In the non-transplant paediatric population, adenovirus infection is the most common cause. The initial infective insult occurs in the early years and the diagnosis requires a history of acute severe bronchiolitis/viral pneumonia in previously healthy children in the first 3 years of life. Though there has been a case report of post-infectious OB in an adult female, ${ }^{1}$ to our knowledge, there are no published cases in children acquired after 3 years of age.

Aim We describe two previously healthy older boys with normal immunological investigations who developed post-infectious OB. Cases The first patient had severe adenovirus pneumonia aged 7 , requiring ventilation, oxygen and bronchodilator therapy. In view of atopic background (nut allergy and paternal asthma) inhaled corticosteroids were initiated at discharge. A year later he presented with productive cough, debilitating shortness of breath on exertion, moderately reduced PEFR but no wheeze.
He did not respond to Amoxicillin or step-up asthma treatments. CXR and exercise test were normal and pulmonary function tests (PFT) did not show evidence of reversibility. A mixed growth of typical respiratory pathogens were isolated in sputum and treated with prolonged oral antibiotic course. High resolution chest CT (HRCT) showed air trapping in the right upper lobe consistent with $\mathrm{OB}$.

The second patient developed severe mixed mycoplasma and adenovirus pneumonia aged 3.5, followed by persistent left lower lobe collapse and wet cough requiring intravenous antibiotics and physiotherapy. His left lower lobe re-expanded, but his HRCT revealed air trapping consistent with OB. He continued to have intermittent chest infections with various typical respiratory pathogens isolated in sputum requiring oral and intravenous antibiotics. His PFTs are stable with moderately reduced FEV1/ FVC.

Both patients are maintained on regular chest physiotherapy and intermittent antibiotics.

Conclusion Post-infectious OB can develop in healthy children older than 3 years.

\section{REFERENCE}

1 Marinopoulos GC, Huddle KR, Wainwright $H$. Obliterative bronchiolitis: virus induced? Chest 1991;99(1):243-5

\section{P92 REAL-TIME ONLINE ANALYSIS OF VOLATILE ORGANIC COMPOUNDS IN THE EXHALED BREATH OF PRESCHOOL CHILDREN}

KA Holden, SF Hussain, D Roland, TJ Coats, EA Gaillard. University of Leicester, Leicester, UK

10.1136/thoraxjnl-2015-207770.229

Introduction Investigating airway inflammation and pathology in preschool children is challenging from both a technical and ethical standpoint and as such we urgently need to identify and validate novel, non-invasive techniques. Analysis of exhaled volatile organic compounds (eVOC) has been successfully employed using conventional, offline mass spectrometry in preschoolers (van de Kant 2013). However, real-time, instant analysis of eVOC in this age group would be an important advance in the field of 'breathomics'. We sought to assess whether real-time online analysis of eVOC was feasible in preschool children attending our hospital.

Methods Breath samples were taken from preschool children (aged between 1 and 6 years) attending the emergency department or an acute medical ward at our hospital. Children breathed room air tidally via a facemask and eVOC were analysed instantly by Proton-Transfer-Reaction time-of-flight mass spectrometry (PTR-Tof-MS) coupled to Loccioni breath collection apparatus. Capnography data was simultaneously recorded. The mass spectra were analysed using a Matlab programme coded for adult patients and the spectra for mass channel $(\mathrm{m} / \mathrm{z})$ 59 (acetone) were inspected. Total counts for all mass channels were summated.

Results Eight children (median age 42 months, range 14-59 months) participated, of which five (median age 40 months) were able to produce analysable results. The total median count from the summation of all mass channels was 157,465 ncsp (number of counts per second) ranging from 4,831 to 200,319 ncps. The Figure 1 below demonstrates a spectrum for $\mathrm{m} / \mathrm{z} 59$ and capnography trace from one of the participants. The patterns of both traces are comparable. 